\title{
Innovación y sostenibilidad en la empresa L*
}

Innovation and sustainability in company $L$

Citar como: Pérez Uribe, R. (2020). Innovación y sostenibilidad en la empresa L. CITAS, 6(1). https://doi.org/10.15332/2 4224529.6365

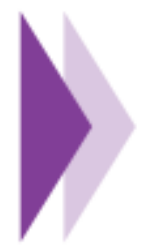

Rafael Pérez Uribe ${ }^{1}$

1 Universidad Santo Tomás

Correo electrónico: ripudos@gmail.com 


\section{Resumen}

El presente artículo tiene como objetivo presentar a la comunidad académica y empresarial la aplicación y análisis del modelo RISE (ruta de innovación y sostenibilidad empresarial) en la empresa L, dedicada a la evaluación en superficie, optimización de la producción e intervención de pozos, a través de la integración y adaptación de los recursos humanos, tecnológicos y financieros, para mantener una participación en el mercado de los hidrocarburos. Este análisis parte de la identificación del comportamiento de los indicadores financieros de la empresa, con el propósito de determinar los niveles de rentabilidad del total de los activos y la obtenida por la organización sobre sus fondos, a partir de datos de los estados financieros. Además, la ejecución del modelo RISE en la empresa sirvió para la identificación de riesgos y fortalezas, mediante un diagnóstico e intervención de los nueve factores y las cuatro dimensiones manejadas en este modelo. Así mismo, se estableció un informe de análisis y se generó un plan de acción que incluyó el grado de prioridad para la ejecución de las actividades, el recurso económico, el personal involucrado y la duración de las actividades de innovación y sostenibilidad. Finalmente, la aplicación del modelo RISE a la empresa L permitió trabajar con el compromiso de la alta gerencia, los líderes de los departamentos y los colaboradores. Esto mediante planes de acción que conllevaron a la mejora continua de la organización.

Palabras clave: RISE, innovación, tecnología, cultura organizacional, nuevos mercados, procesos colaborativos,

En el mundo actual de la economía, las organizaciones se han caracterizado por ser dinámicas, innovadoras y estratégicas, donde al tiempo se espera que su enfoque esté orientado a intereses económicos, sociales, ambientales y gerenciales. Es por esto por lo que día a día se gestionan sus acciones de manera sostenible y la mayoría de las veces se convierte en un proceso fundamentalmente metodológico. También se afirma que el control de una o varias estrategias dependerá en detectar las causas que las desvían para desarrollar la misión y alcanzar la visión hacia donde se dirige cada empresa y reaccionar a tiempo para reorientar las según lo planeado. Para la ejecución de la estrategia es importante definir modelos eficaces y dinámicos que permitan medir la ejecución, el nivel de rendimiento alcanzado por la organización y el grado de implantación de la estrategia diseñada (Arjona, 1999).

Thompson y colaboradores (2012, p. 5), y Pérez-Uribe (2018, p. 92) mencionan que la estrategia de una compañía es el plan de acción o acciones que sigue la administración para competir con éxito y obtener utilidades a partir de un arsenal integrado de opciones, lo cual indica que las organizaciones buscan mantenerse a toda costa para competir satisfactoriamente.

Teniendo en cuenta lo anterior, Pérez-Uribe y Ramírez-Salazar (2019) junto con un equipo de consultores e investigadores de la Universidad EAN crean el modelo RISE (ruta de innovación y sostenibilidad empresarial), que se implementa en las organizaciones con el propósito de mostrar de forma rápida el nivel de madurez en el cual se encuentran frente a la tendencia mundial, así como el camino que se deben tomar para gerenciar las mejores prácticas. Su origen se fundamenta en tres modelos gerenciales y un estándar internacional; 1) el modelo de modernización para la gestión de las organizaciones (Pérez-Uribe et ál., 2013); 2) el modelo de innovación abierta colaborativa (Ramírez-Salazar, 2016); 3) el modelo del design thinking (Razzouk y Shute, 2012); reconocimiento, indicadores financieros, liderazgo, producción sostenible.

\section{Abstract}

This article aims to present to the academic and business community the application and analysis of the RISE model (business innovation and sustainability route, RISE in Spanish) in the company $\mathrm{L} 3 / 4$ dedicated to surface evaluation, production optimization and well intervention $3 / 4$ through the integration and adaptation of human, technological, and financial resources, to maintain a share in the hydrocarbon market. This analysis starts with the identification of the behavior of the company's financial indicators, to determine the profitability levels of the total assets and the profitability obtained by the organization on its funds, based on data from the financial statements. In addition, the implementation of the RISE model in the company served to identify risks and strengths, through a diagnosis and intervention of the nine factors and the four dimensions handled in this model. Likewise, an analysis report was established and an action plan was generated, which included the priority level for the execution of the activities, the economic resources, the personnel involved and the duration of the innovation and sustainability activities. Finally, the application of the RISE model to company L allowed us to work with the commitment of senior management, department leaders and collaborators. This was achieved through action plans that led to continuous improvement of the organization.

Keywords: RISE, innovation, technology, organizational culture, new markets, collaborative processes, recognition, financial indicators, leadership, sustainable production.

y 4) el estándar de medición del Global Reporting Initiative (GRI, 2016; Programa de las Naciones Unidas para el Medio Ambiente [PNUMA], 2016).

En este sentido, estos autores y su equipo de trabajo deciden que el modelo se despliegue con nueve factores, y que para cada uno se analice en las organizaciones con una mirada integral involucrando cuatro dimensiones: 1) la social, que describe el modo de innovar; 2) la ambiental, que desarrolla la metodología de ecodiseño y economía circular; 3) la gerencial, que caracteriza los productos, procesos, gestión, estrategia de mercadeo y el modelo de negocio; y 4) la económica, que describe cuál es la creación de valor y qué tanto esta innovación aporta a la empresa.

La empresa L fue constituida en 1980 con la creación de la empresa M, utilizando el proceso de fundición de precisión (microfusión) para producción de componentes para válvulas industriales. En 1984, se constituyó la V Industria y Comercio de V., unidad volcada para la producción de válvulas industriales como forma de agregar valor a los microfundidos producidos por la empresa M. Nació enfocada en la producción de válvulas industriales para atender al mercado industrial a través de distribuidores y revendedores. En el año 2000 adquirió la empresa Metalúrgica NA (actualmente LMNA), fabricante de válvulas de mayor valor agregado para el sector de petróleo y gas, así permitió aumentar su presencia en el sector hidrocarburos. Estableció como estrategia aumentar su exposición al sector de petróleo y gas, principalmente en Brasil, donde el sector crecía y continúa creciendo a tasas más altas que el propio PIB. Para ello decidió iniciar un proceso de adquisición de nuevos negocios que permitiría traer nuevas tecnologías y acceso a nuevos mercados dentro del sector de petróleo y gas. 
En el 2010 se convierte en L Colombia con la adquisición de HS, empresa colombiana con más de 16 años de operaciones en el país. Actualmente cuenta con operaciones a nivel nacional, desarrolla sus actividades y ofrece sus servicios en las líneas de WT, WL, C, $7 \mathrm{~S}, \mathrm{Ct}$ y frentes de inyección de agua en los distritos norte (Magdalena medio y Santanderes), centro (Meta y alrededores) y sur (Huila y Tolima).

Como un ejercicio académico y empresarial, se aplicó el modelo RISE para medir el nivel de cada factor con sus dimensiones y así formular una ruta que le permitiese a la empresa manejar las mejores prácticas en innovación y sostenibilidad, para mejorar su posición competitiva y atender más de cerca de sus stakeholders.

\section{Breve descripción el modelo RISE}

Con este modelo se evalúan en la organizaciones nueves factores (Pérez-Uribe et ál., 2020): 1) innovación, que consiste en analizar cómo la empresa genera un valor agregado al producto y al servicio mediante procesos, procedimientos sostenibles para satisfacer las necesidades y superar las expectativas de los clientes; 2) producción sostenible, que mira cómo la empresa maneja estratégicamente los recursos naturales y residuos mediante la realización de sus tareas o funciones; 3) liderazgo y direccionamiento estratégico, determina la interacción que tiene la alta dirección con la organización mediante la toma de decisiones para el cumplimiento de los objetivos organizacionales; 4) cultura organizacional, basada en identificar las condiciones que ofrece la empresa para preservar un ambiente laboral adecuado entre sus colaboradores; 5) reconocimiento, que trata sobre los mecanismos que tiene la empresa para reconocer y premiar a sus colaborares por su buen rendimiento dentro de la organización o en la eficacia y eficiencia en la realización de sus tareas mediante incentivos; 6) procesos colaborativos, el cual consiste en identificar las alianzas que tiene la empresa con organizaciones del mismo sector en materia de generar una sinergia entre las dos partes; 7) nuevos mercados, trata sobre el análisis de los planes de marketing que presenta la empresa para incursionar en nuevos segmentos o nichos de mercados; 8) tecnología, que básicamente consiste en observar y analizar si la empresa emplea equipos tecnológicos sostenibles en la realización de sus funciones; y 9) indicadores financieros con los cuales se determina el estado de la empresa en términos de liquidez, rentabilidad y endeudamiento. Además, si está generando ganancia o pérdida neta.

\section{Metodología}

Con base en Hernández-Sampieri, Fernández y Baptista (2010), este trabajo es descriptivo y transversal, ya que los datos se recolectaron en un solo periodo y las variables fueron analizadas e interrelacionadas en un tiempo específico.

El instrumento utilizado para realizar al análisis organizacional fue el Modelo RISE (ruta de innovación y sostenibilidad empresarial), que actualmente se está aplicando por un grupo de expertos relacionados con el tema de innovación y sostenibilidad en los posgrados de varias universidades colombianas. Este modelo cuenta con una matriz en Excel y un software en la web (rise.ean.edu.co), con los cuales se recopila la información con las personas que en las organizaciones manejan cada uno de los factores. Incluye nueve factores y en cada uno se analizan cuatro dimensiones con sus respectivos descriptores: social, ambiental, gerencial y económica (Ramírez-Salazar et ál., 2019, p. 317).

Cada descriptor a su vez tiene cinco niveles de madurez (Ramírez-Salazar et ál., 2019, p. 317): nivel 1: nivel incipiente, en el que existe una relación inexistente o muy baja con el descriptor del factor evaluado; nivel 2: nivel de desarrollo, etapa básica en la que hay una relación parcial o informal con el descriptor del factor evaluado; nivel 3: nivel intermedio, en el que se evidencia que existe un nivel inicial, formal, una manera planificada de trabajar hacia el descriptor del factor evaluado; nivel 4: nivel de madurez, en el que el propietario considera importante seguir trabajando hacia alcanzar el más alto nivel, para posicionar su empresa en la industria como innovadora y sostenible; y nivel 5: nivel de excelencia, en el que la empresa se destaca por encima de otras empresas debido a sus buenas prácticas en innovación y sostenibilidad en el ámbito nacional e internacional.

\section{Hallazgos}

\section{Análisis general de descriptores modelo RISE}

Teniendo presente las cuatro dimensiones: social, ambiental, gerencial y económica, se resalta que los factores con mayor fortaleza en la compañía son: nuevos mercados, cultura organizacional, liderazgo y direccionamiento estratégico y producción sostenible, que cuentan con un porcentaje entre $60 \%$ a $80 \%$. Por otro lado, se resaltan como de alto riesgo estos factores: indicadores financieros, tecnología, procesos colaborativos, reconocimiento e innovación, con porcentajes por debajo del $50 \%$ (figura 1). 
Figura 1. Consolidado factores y dimensiones, empresa L

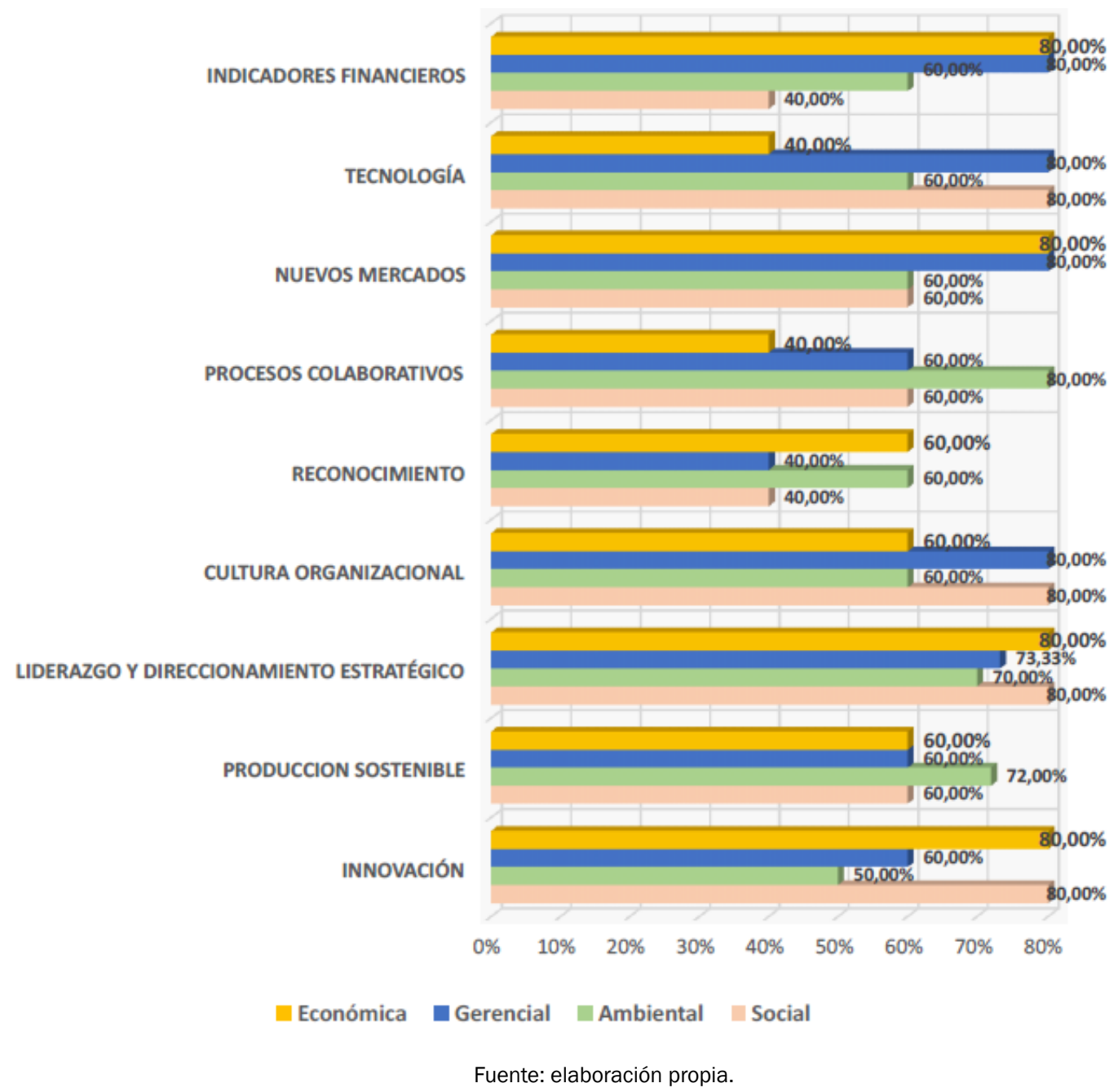

Análisis específico de fortalezas y riesgos por dimensiones Innovación

El factor innovación se ubica en un nivel de normalidad con un 67.5 $\%$, pero se evidencian como fortalezas las dimensiones económica
$(80 \%)$ y social $(80 \%)$. En un nivel normal está la gerencial (60 $\%)$. En riesgo se encuentra la dimensión ambiental (50\%) en los descriptores de ecodiseño y economía circular (figura 2). 
Figura 2. Resultados dimensiones de innovación

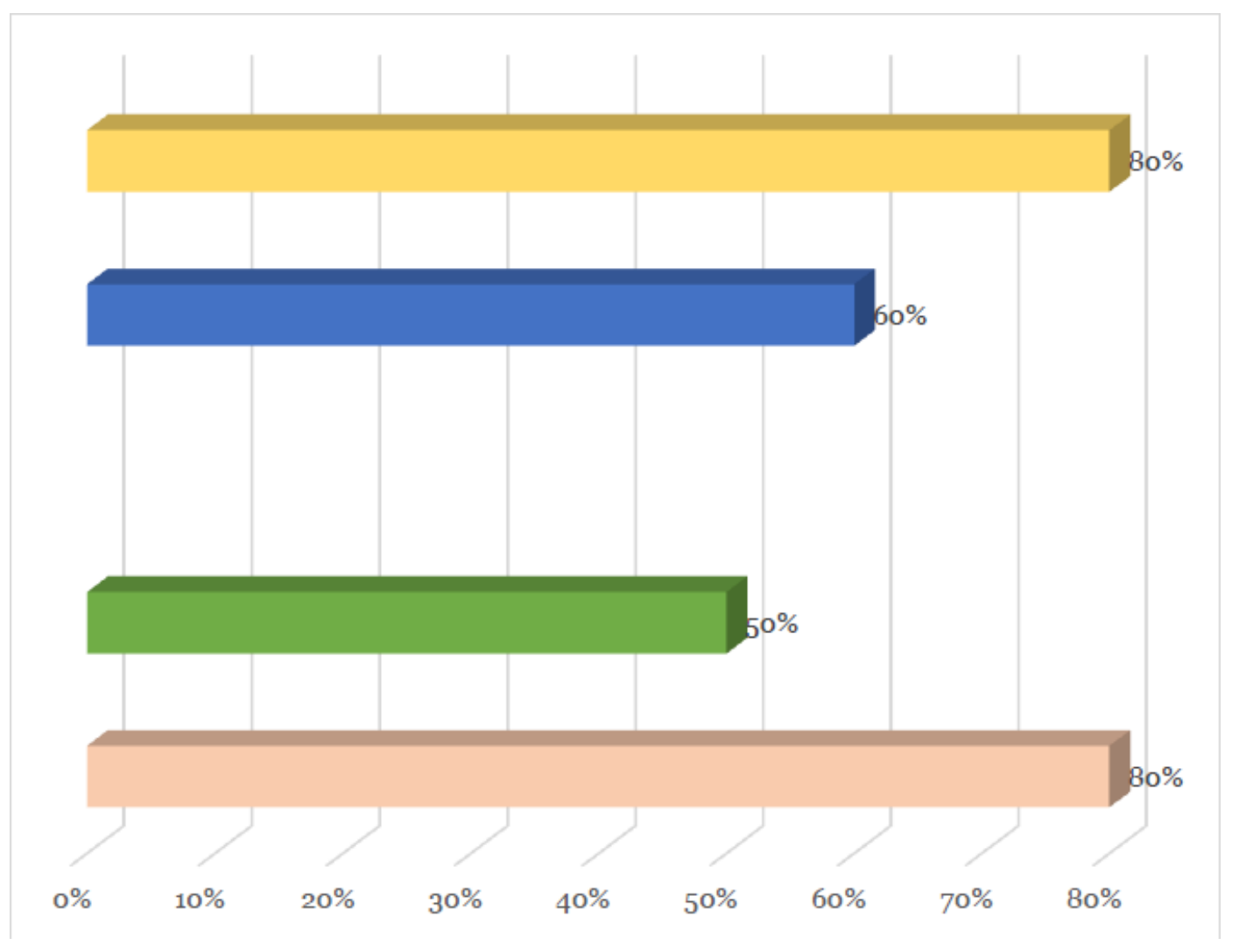

Fuente: elaboración propia.

\section{Producción sostenible}

En este factor se observa que sobresale la dimensión ambiental, con un porcentaje de $72 \%$. Mientras que las dimensiones social, gerencial y económica están en un nivel normal, con el $60 \%$ cada una. Cabe anotar que uno de los descriptores para tener presente en el análisis será incluir el modelo de negocio que incluye economía circular (figura 3). 
Figura 3. Resultados producción sostenible

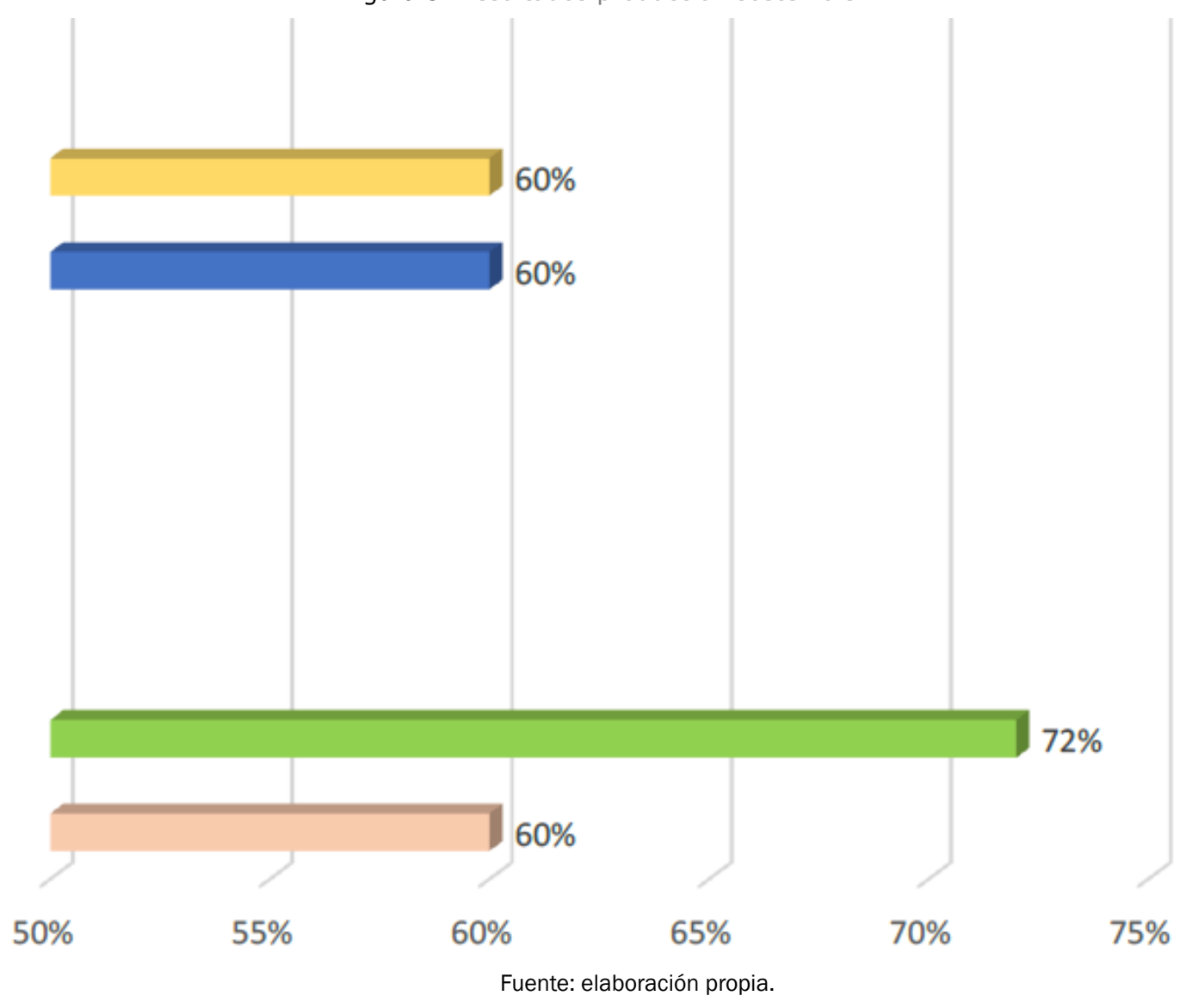

Direccionamiento estratégico y liderazgo

Este factor es uno de los que cuenta con resultados positivos con dimensiones que se encuentran entre un $70 \%$ y $80 \%$. Así mismo se resaltan resultados adecuados dentro de los descriptores del factor (figura 4). 
Figura 4 Resultados direccionamiento estratégico y liderazgo

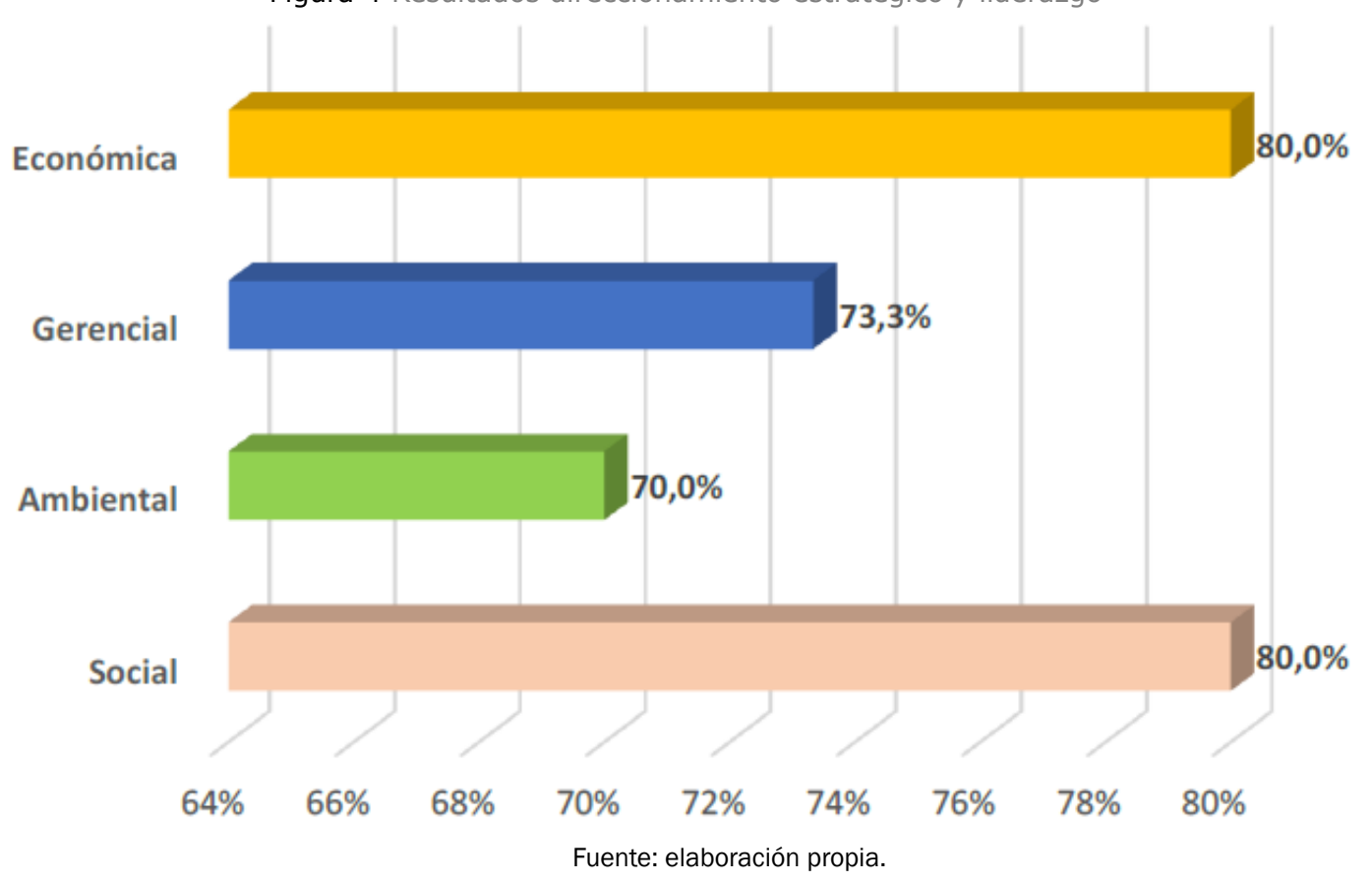

\section{Cultura organizacional}

El factor cultura organizacional cuenta con resultados positivos, donde las dimensiones se encuentran entre un $60 \%$ y $80 \%$; de igual manera se resaltan resultados adecuados dentro de los descriptores del factor (figura 5).

Figura 5. Resultados en las dimensiones del factor cultura organizacional

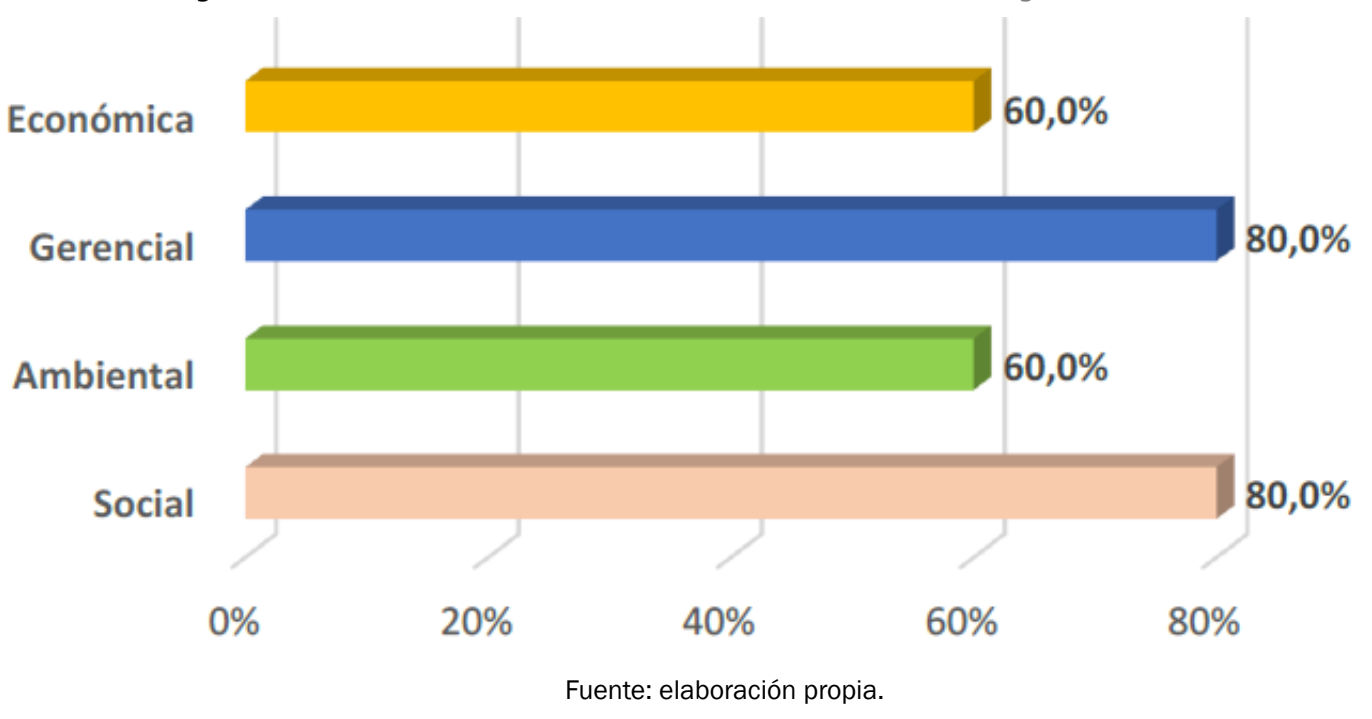


por debajo del $50 \%$, con los descriptores prácticas de motivación y felicidad en el trabajo para tenerlos presente en el momento de definir la ruta de innovación y sostenibilidad para llevarlo a un nivel superior (figura 6).

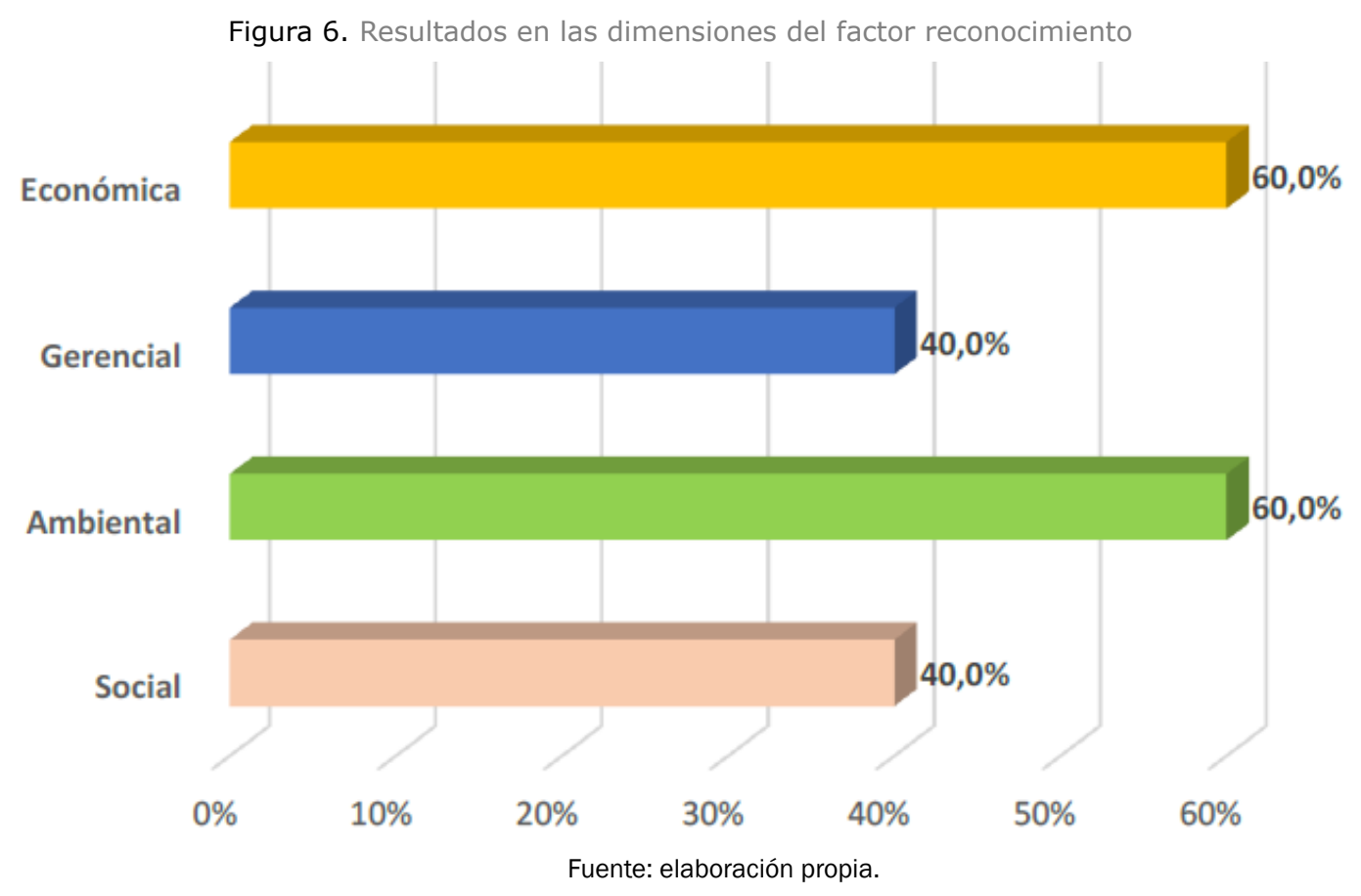

\section{Procesos colaborativos}

Se observa que la dimensión ambiental es una fortaleza, con un $80 \%$, y las dimensiones social y gerencial se encuentran en un nivel normal. Mientras que la dimensión económica en el descriptor de voluntariado corporativo se encuentra en riesgo y requiere de actividades de fortalecimiento para llevarla a niveles superiores (figura 7).

Figura 7. Resultados en las dimensiones del factor procesos colaborativos

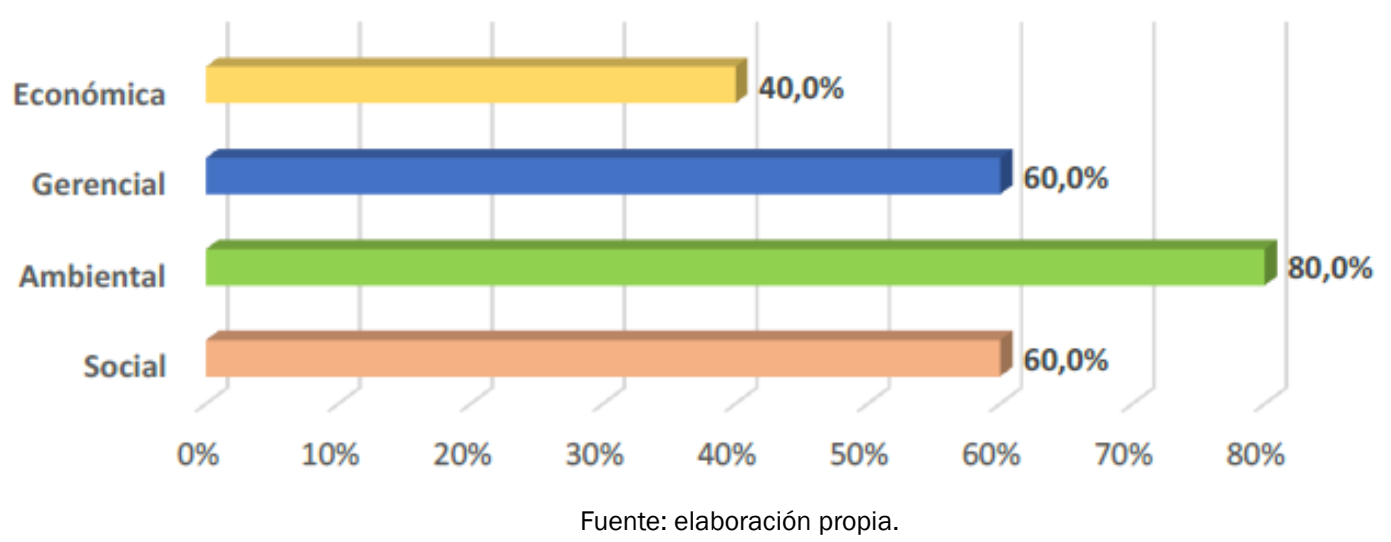

\section{Nuevos mercados}

El factor de nuevos mercados es uno de los que cuenta con resultados positivos, donde las dimensiones económica y gerencial son fortalezas $(80 \%)$ y la social y ambiental están en un nivel normal $(60 \%)$ (figura 8$)$. 
Figura 8. Resultados en las dimensiones del factor nuevos mercados

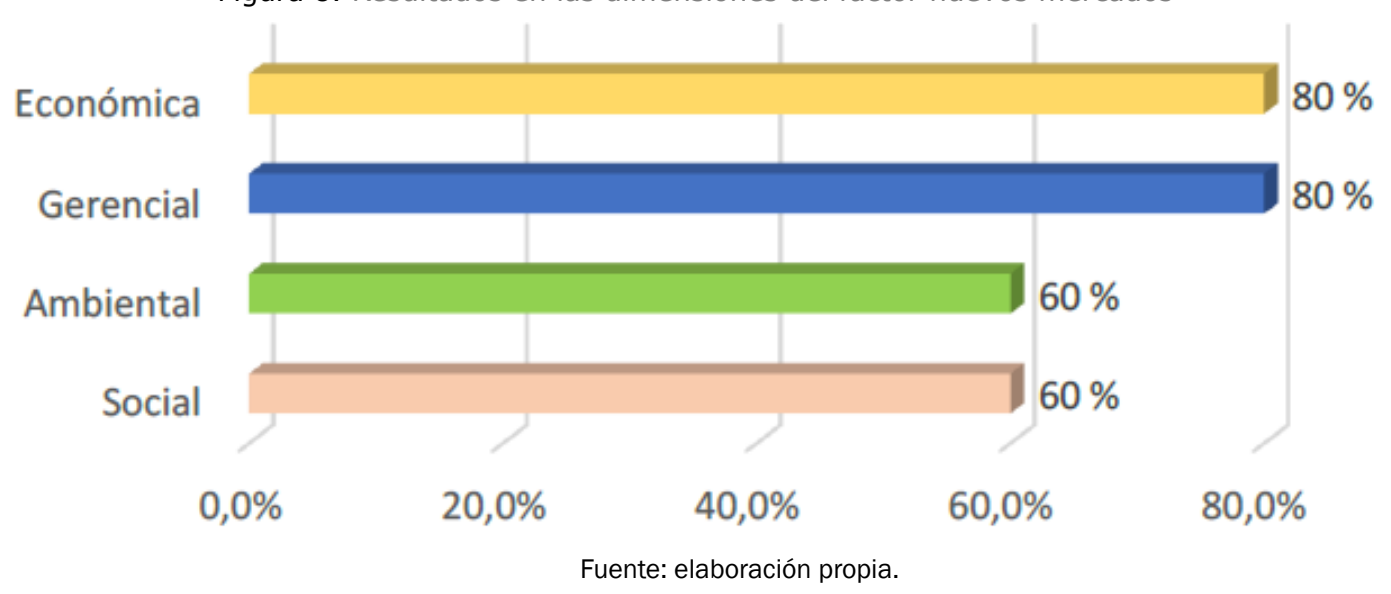

\section{Tecnología}

De las cuatro dimensiones analizadas en la empresa se observa que la económica se encuentra en riesgo, con un $40 \%$. La ambiental se sitúa en normalidad con el $60 \%$, y la ambiental y social son fortalezas con un $80 \%$ cada una (figura 9 ).

Figura 9. Resultados en las dimensiones del factor tecnología

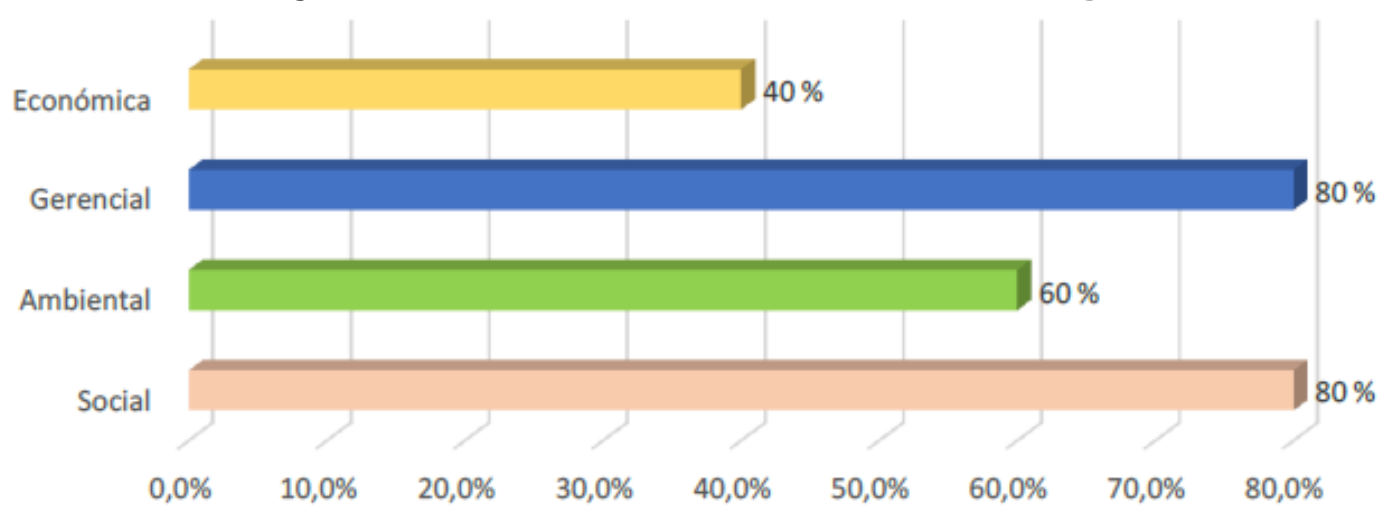

Fuente: elaboración propia.

\section{Indicadores financieros}

El factor de indicadores financieros resalta su fortaleza en la dimensión económica y ambiental, con un $80 \%$ cada una. Mientras que la gerencial se mantiene normal $(60 \%)$ y la social, en riesgo con un $40 \%$ en lo que respecta al impacto en la sociedad (figura $10)$. 
Figura 10. Resultados en las dimensiones del factor indicadores financieros

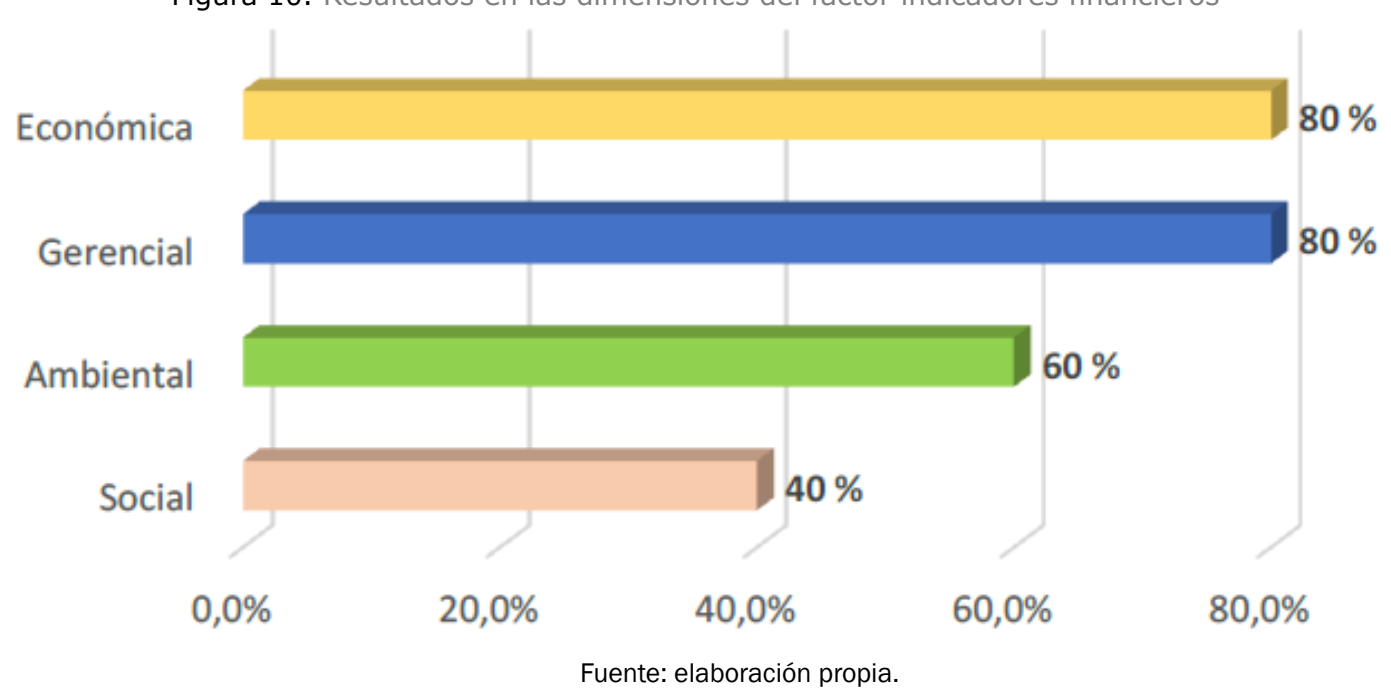

Por otro lado, realizando un análisis flash del comportamiento financiero en miles de COP, se observa lo siguiente:

Para realizar el análisis de los datos financieros de L es necesario tener dos estados financieros a la mano: el balance general o el estado de situación financiera que permite analizar la estructura financiera de la empresa, que está conformada por tres cuentas: activos, pasivos y patrimonio. De este balance se obtienen dos datos: el total de los activos, que es la suma de los activos corrientes y no corrientes de la empresa - entiéndase por activos a todos los recursos, bienes y derechos propios de la empresa que son empleados para la realización de las tareas y de las funciones y el patrimonio, que es el capital social de los accionistas o de los socios- Los demás datos se recogen del estado de resultados que emplea los ingresos, utilidades, costos y gastos para permitir ver de manera detallada el estado del ejercicio en cada año.

Los ingresos operacionales de la empresa están representados por el nivel de ventas que la empresa alcanzó en estos años. Se puede observar que el nivel de ventas en todos los años no fue directamente proporcional, ya que en el año 2016 hubo una baja bastante significativa a COP 30384294 respecto al año 2015. En el año 2017 hubo una recuperación en ventas de COP 53349 088; en el 2018 también hubo un incremento proporcional de COP 73 797572 respecto al 2017.

La utilidad bruta está representada por los ingresos menos el costo de venta de los productos y de los servicios que ofrece la empresa a los clientes. Se puede observar que el año de crisis de la empresa fue el año 2016, ya que su utilidad bruta tuvo un resultado negativo de COP -2 320183 , lo que significa que las ventas en ese año fueron menores a los costos de ventas de los productos o servicios. Respecto al año 2015 el desplome fue significativo, también se puede observar que el año 2017 fue el año de recuperación de esta empresa, ya que la utilidad operacional fue positiva con un valor de COP 8352898 , observando que las ventas fueron superiores a los costos de venta de los productos y servicios.

La utilidad operacional de L está dada por el resultado de los ingresos operacionales menos los costos y gastos operacionales. De igual manera, como pasa en la utilidad bruta, el año 2016 representa un valor negativo en este rubro, con un valor de COP -5 723841 , lo que da a entender que los gastos y los costos operacionales son los que estuvieron directamente relacionados con la razón de ser de la empresa, ya que fueron superiores a los ingresos operacionales, es decir, las ventas generadas por los productos y servicios que ofrecía la empresa a su grupo de clientes potenciales. Se observa que en el año 2015 la utilidad operacional tuvo el pico más alto, con un valor de COP 11068 531. A partir del año 2017 el crecimiento fue directamente proporcional con un valor de COP 5125729 y en el año 2018 fue de COP 9958 059, donde los ingresos operacionales fueron superiores a los costos y gastos.

Los rubros de ganancias y pérdidas de la empresa están determinados por los ingresos menos los gastos. Cuando el valor es positivo son ganancias, lo que determina que en el año 2015 las hubo con un valor de COP 4765433 y en el año 2018 se incrementó la ganancia a COP 7172959 . En el año 2016 y en el año 2017 los datos fueron de carácter negativo es decir que en estos años la empresa generó pérdidas en mayor proporción en el año 2016 con un valor de COP -6 547895 y en el año 2017 se redujeron pérdidas a COP -1852781 .

Los costos hacen referencia a todas las erogaciones que son destinadas para la producción del producto y la prestación de servicios en el sector de los hidrocarburos por parte de la empresa. Como se puede observar, los costos fueron altos en los años 2015 y 2018 con COP 61208432 y con COP 62731 882. En el 2016 los costos fueron bajos, con COP 37214 510, guarismo que evidencia que la producción fue baja por parte de la empresa ante la falta de un flujo de efectivo, que fue ocasionado por las ventas o por los ingresos operacionales que en ese año fueron las más bajas, en tanto que la empresa no tenía capacidad de compra para adquirir productos o brindar servicios de calidad de manera sostenible. En el año 2017 la empresa incrementó sus costos en COP 50121919. Este valor estuvo asociado a que la empresa mejoró sus ventas, por tal razón pudo aumentar de nuevo la producción para ofrecer la cantidad de productos necesaria para satisfacer las necesidades de los clientes.

Los gastos de la empresa son todas las erogaciones destinadas en la distribución, administración, mantenimiento de la empresa; 
es decir, no están relacionados directamente con la producción ni la prestación de los servicios de la empresa. Se observa que los gastos son equilibrados en los años 2016 y 2017, que fueron de COP 3 412816 para el 2016 y de COP 32271169 en el 2017. En el 2018 aumentaron a COP 3860 027. En el 2015 los gastos fueron mayores que los otros años analizados (COP 5778 979).

El ebitda de L está representado por la utilidad antes de impuestos, de depreciación y amortización, y se puede observar que en el año 2015 es semejante a la del año 2018 respectivamente de COP 7503976 y de COP 7758 809, lo que indica que la empresa tenía una viabilidad positiva en su proyecto en cuanto a flujo de dinero para inversión o el pago de deudas que tuviera la empresa. En el año 2016 la viabilidad era negativa con un valor de COP -9 467 456, lo que significa que no había una utilidad sino pérdidas, como también se ve en el año 2017 con un valor de COP -180 131, un año caracterizado por la recuperación de L.

En la tabla 1 se muestran los datos de la empresa L, desde el año 2015 hasta el año 2018, recopilados del balance general y del estado de resultados.

Tabla 1. Estados financieros empresa $L$

\begin{tabular}{|c|c|c|c|c|}
\hline $\begin{array}{l}\text { Estados financieros } \\
\text { cortados a } 31 \text { diciembre, } \\
\text { en miles de pesos } \\
\text { colombianos (\$) }\end{array}$ & 2015 & 2016 & 2017 & 2018 \\
\hline \multicolumn{5}{|c|}{ Información inicial (ingrese en estos espacios los datos solicitados) } \\
\hline Total activos & $\$ 94280199$ & $\$ 68232235$ & $\$ 70761104$ & $\$ 73949457$ \\
\hline Patrimonio & $\$ 50870227$ & $\$ 44322332$ & $\$ 48443201$ & $\$ 55616159$ \\
\hline Ingresos operacionales & $\$ 72209885$ & $\$ 30384294$ & $\$ 53349088$ & $\$ 73797572$ \\
\hline Utilidad bruta & $\$ 15384944$ & $-\$ 2320183$ & $\$ 8352898$ & $\$ 13818086$ \\
\hline Utilidad operacional & $\$ 11068531$ & $-\$ 5723841$ & $\$ 5125729$ & $\$ 9958059$ \\
\hline Ganancias y pérdidas & $\$ 4765433$ & $-\$ 6547895$ & $-\$ 1852791$ & $\$ 7172959$ \\
\hline Costos & $\$ 61208432$ & $\$ 37214510$ & $\$ 50121919$ & $\$ 62731882$ \\
\hline Gastos & $\$ 5778979$ & $\$ 3412816$ & $\$ 3227169$ & $\$ 3860027$ \\
\hline Ebitda & $\$ 7503976$ & $-\$ 9467456$ & $-\$ 180131$ & $\$ 7758809$ \\
\hline
\end{tabular}

Fuente: Superintendencia de Sociedades (s. f).

A continuación, en la tabla 2 y figura 11, se observa el perfil financiero de $\mathrm{L}$, donde se presentan los principales indicadores financieros el ROA, ROE, margen bruto, margen neto, margen operacional y margen ebitda de los años 2015 al 2018.

El primer indicador que se observa es el retorno sobre activos (ROA), que mide la rentabilidad (la utilidad bruta) sobre el total de los activos. Se observa que el valor de este indicador en el año 2015 fue de $11.74 \%$, muy semejante al del año 2018 con un valor de $13.47 \%$. En el año 2016 el valor es negativo con un valor de -8.39 $\%$ mientras que en el año 2017 hubo una recuperación a un $7.24 \%$. Este análisis evidencia el uso eficiente en la gestión de los activos en función de generar beneficios futuros por parte de la empresa, a excepción del año 2016, cuyo valor fue negativo dado que estaba pasando por una crisis económica.

El segundo indicador es el retorno sobre el capital (RSC) o el (ROE) que mide las ganancias y las pérdidas sobre el patrimonio total. Se observa que en la empresa que en el año 2015 el valor fue de $9.37 \%$, y cayó abruptamente en el año 2016 con un valor de $-14.77 \%$. Para ese momento la capacidad de la empresa para remunerar a sus accionistas era nula sobre recursos propios. En el año 2017 el ROE volvió a caer al $-3.82 \% \%$ con respecto al año 2016 y en el 2018 también volvió recuperarse a un $12.90 \%$. Esto conllevó a que el retorno de los recursos propios invertidos creciera proporcionalmente a partir del año 2016.
En el siguiente indicador, el margen neto que parte de las ganancias y pérdidas sobre los ingresos operacionales, se observa una baja bastante significativa del año 2015 de $6.60 \%$ y al año 2016 de $-21.55 \%$. Este resultado se debe al nivel tan bajo de ventas que tuvo la empresa en el año 2016. Como el nivel de ventas aumentó significativamente entre el año 2017 y 2018, el margen neto aumentó de $-3.47 \%$ a $9.72 \%$, respectivamente.

Acompañado de este indicador se presenta el margen bruto, que parte de la utilidad bruta sobre los ingresos operacionales. En el año 2015 el indicador fue de $21.31 \%$, pero en el 2016 se redujo a $-7.64 \%$. Este factor también se vio afectado por el nivel de ventas que tuvo la empresa en ese año, que fue de COP 30384 294, cifra realmente baja en relación con los niveles de ventas de los años 2015 y 2018. En el año 2017 el indicador se recuperó en un 23.3 $\%$ respecto al año 2016, y en el 2018 aumentó en un $3.06 \%$ tras llegar a $18.72 \%$

Posteriormente viene el indicador de margen operacional, que está dado por la utilidad operacional sobre los ingresos operacionales. En el año 2015 el valor fue de $15.33 \%$, pero en el año 2016 el resultado bajó (-18.84 \%), con una diferencia de -34.17 $\%$, que también se vio afectado por el nivel de ventas de ese año. En el 2017, como el nivel de ventas o de ingresos operacionales fue de COP 53349 088, el indicador pasó a $9.61 \%$, en tanto que 
en el 2018 las ventas fueron de COP 73797 572, lo que afectó de manera positiva con un ascenso de $13.59 \%$.

Finalmente, en el margen ebitda, que está dado por el ebitda sobre los ingresos operacionales, se puede determinar que en el año 2015 fue de $10.39 \%$. Para 2016 el nivel de ingresos bajó a COP 41825591 con respecto al año 2015, lo que afectó el indicado a
$-31.159 \%$. En el año 2017 el nivel de ingresos operacionales se incrementó en COP 22964794 y el indicador migró a - 0.33 \% con comportamiento negativo. Pero la empresa se estaba recuperando ante la crisis que había tenido que afrontar. En el 2018 el indicador de las ventas se apuntaló en COP 20448484 y el indicador se ubicó en $10.51 \%$, dato semejante al del año 2015 .

Tabla 2. Indicadores financieros $L$

\begin{tabular}{|c|c|c|c|c|}
\hline Cifras de negocio e indicadores & 2015 & 2016 & 2017 & 2018 \\
\hline Retorno sobre activos (ROA) & 11.74 & -8.39 & 7.24 & 13.47 \\
\hline $\begin{array}{l}\text { Retorno sobre el capital (RSC) (ROE - return } \\
\text { on equity) }\end{array}$ & 9.37 & -14.77 & -3.82 & 12.90 \\
\hline Margen neto & 6.60 & -21.55 & -3.47 & 9.72 \\
\hline Margen bruto & 21.31 & -7.64 & 15.66 & 18.72 \\
\hline Margen operacional & $15 \cdot 33$ & -18.84 & 9.61 & 13.49 \\
\hline Margen ebitda & 10.39 & -31.159 & -0.3376 & 10.5136 \\
\hline
\end{tabular}

Fuente: elaboración propia.

Figura 11. Indicadores financieros 2015-2018

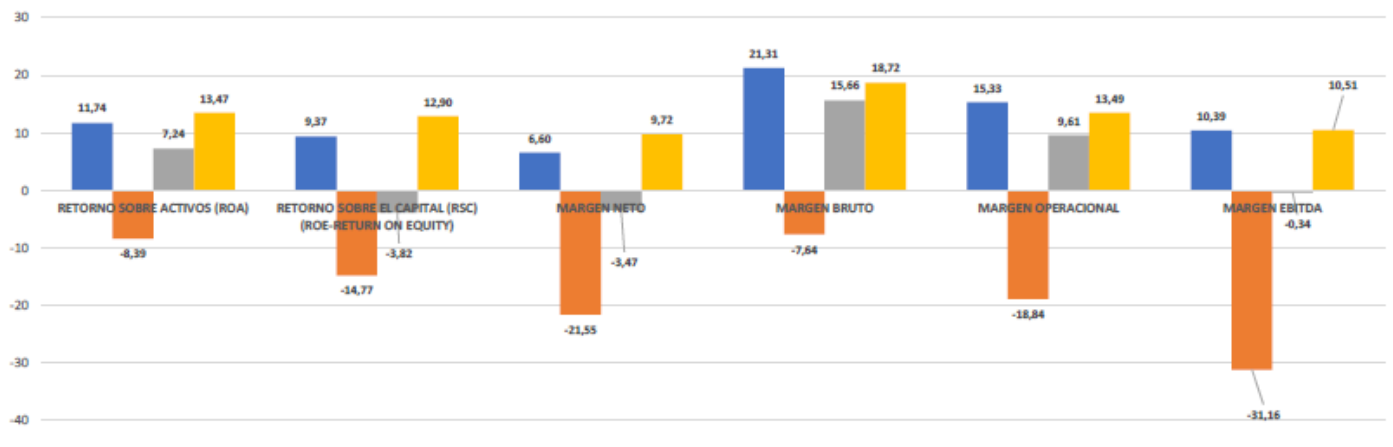

| 2015 | $2016 \mid=2017=2018$

Fuente: elaboración propia.

\section{Conclusiones y recomendaciones}

Esta sección hace referencia directa a lo que debe realizar la empresa $L$ para mejorar aquellos descriptores que se encontraron en riesgo, de acuerdo con los hallazgos descritos en el punto anterior Se despliega a continuación el plan propuesto a la gerencia de la empresa (tabla 3 ) y el respectivo cronograma a corto plazo (tabla 4). 
Tabla 3. Ruta de innovación y sostenibilidad para la empresa L

\begin{tabular}{|c|c|c|c|c|c|c|c|}
\hline \multirow{2}{*}{ Componentes } & \multirow{2}{*}{ Variables } & \multirow{2}{*}{ Actividades } & \multirow{2}{*}{ Prioridad } & \multicolumn{2}{|c|}{ Recursos } & \multirow{2}{*}{$\begin{array}{c}\text { Duración } \\
\text { (meses) }\end{array}$} & \multirow{2}{*}{$\begin{array}{c}\text { Líder } \\
\text { proyecto }\end{array}$} \\
\hline & & & & $\$$ & Humanos & & \\
\hline \multirow[t]{3}{*}{ Cultura organizacional } & $\begin{array}{l}\text { Participación y } \\
\text { compromiso }\end{array}$ & $\begin{array}{l}\text { Definir espacios de } \\
\text { participación que } \\
\text { fomenten la } \\
\text { autonomía de los } \\
\text { trabajadores } \\
\text { dentro de su lugar } \\
\text { de trabajo, a partir } \\
\text { de una escucha } \\
\text { activa y respuesta } \\
\text { oportuna de sus } \\
\text { iniciativas. Así } \\
\text { como el desarrollo } \\
\text { de indicadores que } \\
\text { permitan su } \\
\text { medición. }\end{array}$ & 1 & 0 & 378 & 1 & Gerente TH \\
\hline & \multirow{2}{*}{$\begin{array}{l}\text { Desarrollo y } \\
\text { reconocimiento }\end{array}$} & $\begin{array}{l}\text { Desarrollar } \\
\text { programa para } \\
\text { preparar a las } \\
\text { personas que se } \\
\text { acercan al retiro } \\
\text { laboral. }\end{array}$ & 4 & $\circ$ & 2 & 12 & \\
\hline & & $\begin{array}{l}\text { Definir un plan de } \\
\text { reconocimiento a } \\
\text { partir de unos } \\
\text { criterios y un } \\
\text { sistema de puntos } \\
\text { establecidos para }\end{array}$ & 2 & $\$ 5000000$ & 4 & 3 & \\
\hline
\end{tabular}

Fuente: elaboración propia. 
Tabla 3. Ruta de innovación y sostenibilidad para la empresa L

\begin{tabular}{|c|c|c|c|c|c|c|c|}
\hline \multicolumn{8}{|c|}{ Plan de acción $\mathrm{L}$} \\
\hline \multirow{2}{*}{ Componentes } & \multirow{2}{*}{ Variables } & \multirow{2}{*}{ Actividades } & \multirow{2}{*}{ Prioridad } & \multicolumn{2}{|c|}{ Recursos } & \multirow{2}{*}{$\begin{array}{l}\text { Duración } \\
\text { (meses) }\end{array}$} & \multirow{2}{*}{$\begin{array}{c}\text { Líder } \\
\text { proyecto }\end{array}$} \\
\hline & & & & s & Humanos & & \\
\hline \multirow{5}{*}{ Gestión ambiental } & & $\begin{array}{l}\text { los individuos y } \\
\text { grupos que } \\
\text { contribuyen al } \\
\text { cumplimiento de } \\
\text { los objetivos } \\
\text { organizacionales. }\end{array}$ & & & & & \multirow{5}{*}{$\begin{array}{l}\text { Jefe } \\
\text { HSEQ }\end{array}$} \\
\hline & $\begin{array}{l}\text { Creación de un } \\
\text { entorno vital para } \\
\text { todos los trabajadores }\end{array}$ & $\begin{array}{l}\text { Establecer método } \\
\text { para analizar y dar } \\
\text { respuesta concreta } \\
\text { a los sentimientos, } \\
\text { emociones y estrés } \\
\text { generados por el } \\
\text { entorno. }\end{array}$ & 3 & o & 4 & 1 & \\
\hline & $\begin{array}{l}\text { Sistema de gestión } \\
\text { ambiental }\end{array}$ & $\begin{array}{l}\text { Definir indicadores } \\
\text { de programas } \\
\text { ambientales } \\
\text { asociados con los } \\
\text { análisis de } \\
\text { impactos } \\
\text { ambientales } \\
\text { significativos } \\
\text { (AIAS) y aguas } \\
\text { residuales. }\end{array}$ & 3 & $\circ$ & 1 & 4 & \\
\hline & Residuos sólidos & $\begin{array}{l}\text { Ejecutar programa } \\
\text { posconsumo de los } \\
\text { residuos solidos. }\end{array}$ & 2 & $\circ$ & 2 & 6 & \\
\hline & $\begin{array}{l}\text { Departamento de } \\
\text { gestión ambiental } \\
\text { (DGA) }\end{array}$ & $\begin{array}{l}\text { Establecer el DGA, } \\
\text { donde se } \\
\text { documente sus } \\
\text { procesos y se } \\
\text { registre ante la }\end{array}$ & 4 & $\$ 180000000$ & 4 & 12 & \\
\hline
\end{tabular}

Fuente: elaboración propia.

Tabla 3. Ruta de innovación y sostenibilidad para la empresa L

\begin{tabular}{|c|c|c|c|c|c|c|c|}
\hline \multicolumn{8}{|c|}{ Plan de acción L } \\
\hline \multirow{3}{*}{ Componentes } & \multirow{2}{*}{ Variables } & \multirow{2}{*}{ Actividades } & \multirow{2}{*}{ Prioridad } & \multicolumn{2}{|c|}{ Recursos } & \multirow{2}{*}{$\begin{array}{c}\text { Duración } \\
\text { (meses) }\end{array}$} & \multirow{2}{*}{$\begin{array}{c}\text { Líder } \\
\text { proyecto }\end{array}$} \\
\hline & & & & $\$$ & Humanos & & \\
\hline & & $\begin{array}{l}\text { autoridad } \\
\text { competente. }\end{array}$ & & & & & \\
\hline \multirow{3}{*}{$\begin{array}{l}\text { Innovación y } \\
\text { conocimiento }\end{array}$} & Tipos de innovación & $\begin{array}{l}\text { Formular un } \\
\text { programa de } \\
\text { innovación } \\
\text { sistemática } \\
\text { constituido por } \\
\text { procesos } \\
\text { productivos para } \\
\text { mejorar la } \\
\text { competitividad y } \\
\text { sostenibilidad de la } \\
\text { compañía. }\end{array}$ & 2 & $\$ 200000000$ & 5 & 2 & \multirow{3}{*}{$\begin{array}{l}\text { Gerente TH } \\
\text { Y G. general }\end{array}$} \\
\hline & $\begin{array}{l}\text { Cultura de innovación } \\
\text { sostenible }\end{array}$ & \multirow{2}{*}{$\begin{array}{l}\text { Plantear estímulos } \\
\text { de reconocimientos } \\
\text { para los procesos } \\
\text { de innovaciones, } \\
\text { buscando } \\
\text { implementarlos y } \\
\text { ser reconocidos } \\
\text { como empresa del } \\
\text { sector líder de } \\
\text { innovación. }\end{array}$} & \multirow[b]{2}{*}{1} & \multirow[b]{2}{*}{$\$ 5000000$} & \multirow[b]{2}{*}{4} & \multirow[b]{2}{*}{3} & \\
\hline & $\begin{array}{l}\text { Actores de la } \\
\text { innovación }\end{array}$ & & & & & & \\
\hline
\end{tabular}

Fuente: elaboración propia. 
Tabla 4. Cronograma de trabajo RISE

\begin{tabular}{|c|c|c|c|c|c|c|c|c|c|c|c|c|c|c|}
\hline \multicolumn{15}{|c|}{ Cronograma ruta de mejoramiento e innovación } \\
\hline Actividades por descriptor & Prioridad & Duración en meses & 1 & 2 & 3 & 4 & 5 & 6 & 7 & 8 & 9 & 10 & 11 & 12 \\
\hline $\begin{array}{l}\text { Definir espacios de participación } \\
\text { que fomenten la autonomía de los } \\
\text { trabajadores dentro de su lugar de } \\
\text { trabajo, a partir de una escucha } \\
\text { activa y respuesta oportuna de sus } \\
\text { iniciativas. Asi como el desarrollo de } \\
\text { indicadores que permitan su } \\
\text { medición. }\end{array}$ & 1 & 1 & $\checkmark$ & & & & & & & & & & & \\
\hline $\begin{array}{l}\text { Desarrollar programa para preparar } \\
\text { a las personas que se acercan al } \\
\text { retiro laboral. }\end{array}$ & 4 & 12 & $\checkmark$ & $\checkmark$ & $\checkmark$ & $\checkmark$ & $\checkmark$ & $\checkmark$ & $\checkmark$ & $\checkmark$ & $\checkmark$ & $\checkmark$ & $\checkmark$ & $\checkmark$ \\
\hline $\begin{array}{l}\text { Definir un plan de reconocimiento a } \\
\text { partir de unos criterios y un sistema } \\
\text { de puntos establecidos para los } \\
\text { individuos y grupos que } \\
\text { contribuyen al cumplimiento de los } \\
\text { objetivos organizacionales. }\end{array}$ & 2 & 3 & $\checkmark$ & $\checkmark$ & & & & & & & & & & \\
\hline $\begin{array}{l}\text { Establecer método para analizar y } \\
\text { dar respuesta concreta a los } \\
\text { sentimientos, emociones y estrés } \\
\text { generados por el entorno. }\end{array}$ & 3 & 1 & $\checkmark$ & & & & & & & & & & & \\
\hline $\begin{array}{l}\text { Definir indicadores de programas } \\
\text { ambientales asociados con los AIAS, } \\
\text { aguas residuales. }\end{array}$ & 3 & 4 & $\checkmark$ & $\checkmark$ & $\checkmark$ & $\checkmark$ & & & & & & & & \\
\hline $\begin{array}{l}\text { Ejecutar programa posconsumo de } \\
\text { los residuos sólidos }\end{array}$ & 2 & 6 & $\checkmark$ & $\checkmark$ & $\checkmark$ & $\checkmark$ & $\checkmark$ & $\checkmark$ & & & & & & \\
\hline
\end{tabular}

Fuente: elaboración propia.

Tabla 4. Cronograma de trabajo RISE

\begin{tabular}{|c|c|c|c|c|c|c|c|c|c|c|c|c|c|c|}
\hline \multicolumn{15}{|c|}{ Cronograma ruta de mejoramiento e innovación } \\
\hline Actividades por descriptor & Prioridad & Duración en meses & 1 & 2 & 3 & 4 & 5 & 6 & 7 & 8 & 9 & 10 & 11 & 12 \\
\hline $\begin{array}{l}\text { Establecer el DGA, donde se } \\
\text { documente sus procesos y se } \\
\text { registre ante la autoridad } \\
\text { competente. }\end{array}$ & 4 & 12 & $\checkmark$ & $\checkmark$ & $\checkmark$ & $\checkmark$ & $\checkmark$ & $\checkmark$ & $\checkmark$ & $\checkmark$ & $\checkmark$ & $\checkmark$ & $\checkmark$ & $\checkmark$ \\
\hline $\begin{array}{l}\text { Formular un programa de } \\
\text { innovación sistemática constituida } \\
\text { por procesos productivos para } \\
\text { mejorar la competitividad y } \\
\text { sostenibiliad de la compañía. }\end{array}$ & 2 & 2 & $\checkmark$ & $\checkmark$ & & & & & & & & & & \\
\hline $\begin{array}{l}\text { Plantear estímulos de } \\
\text { reconocimientos para los procesos } \\
\text { de innovaciones, buscando } \\
\text { implementarlos y ser reconocidos } \\
\text { como empresa del sector líder de } \\
\text { innovación. }\end{array}$ & 1 & 3 & $\checkmark$ & $\checkmark$ & $\checkmark$ & & & & & & & & & \\
\hline
\end{tabular}

Fuente: elaboración propia.

\section{Referencias}

Arjona, M. (1999). Dirección estratégica un enfoque práctico: principios y aplicaciones de gestión de rendimiento. Díaz de Santos.
Global Reporting Initiative (GRI). (2016). GRI 103: Management Approach 2016. https://www.globalreporting.org/standards/me dia/1441/spanish-gri-103-management-approach-2016.pdf

Hernández-Sampieri, R., Fernández, C. y Baptista, MdP. (2010). Metodología de la investigación. (5. a ed.). McGraw-Hill. 
Pérez-Uribe, R., Ocampo-Guzmán, D., Moscoso-Durán, F. y Ramírez-Salazar, M. (2020). Innovation and sustainability management as a key factor in global purpose-driven micro, small, and medium-sized bogotanas companies: its impact on financial results in MSMEs. Chapter 19. En R. Pérez-Uribe (ed.), Handbook of Research on International Business and Models for Global Purpose-Driven Companies (pp. 354-379). IGI Global. https://doi.org/10.4018/978-1-7998-4909-4

Pérez-Uribe, I. y Ramírez-Salazar, M. (2019). Modelo RISE: Ruta de innovación y sostenibilidad empresarial (RISE) [tesis de maestría, Universidad EAN]. Repositorio Universidad EAN. https://repository.ean.edu.co/bitstream/handle/10882/98 66/JaramilloOscar2020.pdf;jsessionid=12119BCE70B4D43D 88D0F5269A92F663? sequence $=1$

Pérez-Uribe, R. (2018). Gerencia estratégica corporativa. Ediciones Ecoe Ltda.

Pérez-Uribe, R., Nieto, M., Velázquez, A., Castellanos, G., Garzón, M., Vargas, H. A., Alfonso, N., Calixto, N., Rodríguez, A., Palacio, M., López, L., Vidal, M. y López, J. (2013). Modelo de Modernización para la Gestión de Organizaciones (MMGO). Universidad EAN.

Secretaría del Ozono. Programa de las Naciones Unidas para el Medio Ambiente (PNUMA). (2016). Manual del Convenio de Viena para la protección de la capa de Ozono. https://observatoriop10.cepal.org/sites/default/files/doc uments/treaties/vc-handbook-2016-spanish.pdf

Superintendencia de Sociedades. (s. f.). Estudios financieros. https://www.supersociedades.gov.co/delegatura_a ec/estudios_financieros/Paginas/sirem.aspx

Ramírez-Salazar, M., Pérez-Uribe, R., Salcedo-Pérez, C. y Juffington-Smith, J. P. (2019). RISE Model: Its Application on Diving Enterprises Located in the San Andres Archipelago (Colombia). En Saiz-Álvarez J., Leitão J. y Palma-Ruiz J. (eds.), Studies on Entrepreneurship, Structural Change and Industrial Dynamics (pp. 327-34). Springer. https://doi.org/10.1007/9783-030-15526-1

Ramírez-Salazar, M. P. (2016). Modelo de innovación abierta colaborativa para la banca de fomento: caso Bancóldex. [tesis doctoral, Universidad EAN]. Biblioteca Universidad EAN. http://edicionesean.ean.edu.co/index.php/productos-de-i nvestigacion 1/tesis-doctorales/23-publicaciones/472-modelo-d e-innovacion-abierta-colaborativa-para-la-banca-de-fomento-c aso-bancoldex

Razzouk, R. y Shute, V. (2012). What is design thinking and why is it important? Review of Educational Research. 82(3), 330-348. https://doi.org/10.3102/0034654312457429

Thompson, A., Peteraf, M., Gamble, J., y Strickland, A. (2012). Administración estratégica. Teoría y casos (18.. ed.). Mc Graw Hill.

Notas

* Nombre ficticio asignado a la empresa para proteger sus datos confidenciales. Agradecimientos a los maestrantes Tania Yisela Nieto Charry y Cristian Santiago Quecán Herrera, quienes cursaron la asignatura de Dirección y gestión empresarial en la maestría en Gestión del Talento Humano de la Universidad Santo Tomás en Bogotá (abril de 2020). 